\title{
A CENTRAL LIMIT THEOREM FOR A CLASS OF $d$-DIMENSIONAL RANDOM MOTIONS WITH CONSTANT SPEED ${ }^{1}$
}

\author{
BY LUIS G. GOROSTIZA
}

Communicated by Harry Kesten, February 7, 1972

Consider the motion of a point in a $d$-dimensional Euclidean space $R^{d}(d \geqq 1)$, which starts out from the origin at the time zero in a random direction with constant speed, and at the end of a random time starts afresh from its position with a direction which is a random orthogonal transformation of the previous direction, and so on for all time. The position of the point in time defines a $d$-dimensional random process with continuous trajectories.

Consider a sequence of such processes; specifically, for $n=1,2, \ldots$,

$$
\begin{aligned}
X_{n}(t)=c_{n} \sum_{i=1}^{N_{n}(t)} \tau_{n, i} & A_{n, i-1} \cdots A_{n, 0} \xi_{n} \\
& +c_{n}\left(t-\sum_{i=1}^{N_{n}(t)} \tau_{n, i}\right) A_{n, N_{n}(t)} \cdots A_{n, 0} \xi_{n}, \quad t \geqq 0,
\end{aligned}
$$

where $c_{n}$ is a positive constant (the speed); $\tau_{n, i}, i \geqq 1$, are nonnegative, nonidentically zero random variables (the times between direction changes); $N_{n}(t)=\max \left\{k: \sum_{i=1}^{k} \tau_{n, i} \leqq t\right\}, t \geqq 0 ; A_{n, i}, i \geqq 1$, are random orthogonal matrices (the changes of direction); $A_{n, 0}=I$; and $\xi_{n}$ is a random unit vector (the initial direction). For each $n$ we assume that $\tau_{n, i}, i \geqq 1$, $A_{n, i}, i \geqq 1$, and $\xi_{n}$ are independent, and that $\tau_{n, i}, i \geqq 1$, are identically distributed, as are $A_{n, i}, i \geqq 1$. I denotes the identity matrix.

To obtain an invariance principle for such sequences of processes having a Gaussian limit we make the following further assumptions. In order that the times between direction changes tend to zero as $n \rightarrow \infty$, we suppose there are random variables $\tau_{i}$ and positive constants $b_{n}$ such that $\tau_{n, i}=\tau_{i} / b_{n}$ and $\lim _{n \rightarrow \infty} b_{n}=\infty$. To prevent the point from remaining on a proper subspace of $R^{d}, d \geqq 2$, or from traveling in only one direction in $R^{1}$, we require the random orthogonal matrices $A_{n, i}$ to be irreducible, the definition of an irreducible random matrix of order $d$ being that it has no almost surely invariant nontrivial subspace if $d \geqq 2$, and that it is not

AMS 1970 subject classifications. Primary 60F05; Secondary 60K99.

Key words and phrases. $d$-dimensional random process, Gaussian process, weak convergence.

${ }^{1}$ This result is contained in the author's $1972 \mathrm{Ph} . \mathrm{D}$. dissertation at the University of California at Los Angeles, written under Professor Charles J. Stone. This research was supported by the Instituto Politécnico Nacional and the Comisión Nacional de Energía Nuclear, Mexico. 
almost surely nonnegative if $d=1$. It is a fact that if $A$ is an irreducible random orthogonal matrix the matrix $I-E A$ is nonsingular. We then have the following functional central limit theorem.

THEOREM. If

(i) $E \tau_{1}^{\alpha}<\infty$ for some $\alpha>3$,

(ii) all weak limit points of $\left\{A_{n, 1}, n \geqq 1\right\}$ are irreducible and

exists, and

$$
\lim _{n \rightarrow \infty}\left(I-E A_{n, 1}\right)^{-1}
$$

(iii) $a_{n}$ are positive constants such that $\lim _{n \rightarrow \infty} c_{n} / a_{n} b_{n}^{1 / 2}=1$, then as $n \rightarrow \infty$ the sequence $\left\{X_{n} / a_{n}\right\}$ converges weakly to a Gaussian random process $X$ with stationary and independent increments, mean zero, and covariance matrix function $\operatorname{EX}(t) X(t)^{\prime}=t C / d E \tau_{1}$, with

$$
C=\operatorname{Var} \tau_{1} I+\left(E \tau_{1}\right)^{2}\left\{\lim _{n \rightarrow \infty}\left[\left(I-E A_{n, 1}\right)^{-1}+\left(I-E A_{n, 1}^{\prime}\right)^{-1}\right]-I\right\}
$$

(the prime denotes matrix transposition).

The proof of the theorem is very long; together with some special cases it will appear elsewhere. Here we will make a few remarks about the limit process and mention some of the main elements of the demonstration.

The limit process may be degenerate, which requires that $\tau_{1}$ be almost surely constant. However, for $d \geqq 3$, we have rank $C \geqq 2$ and weak limit points of $\left\{A_{n, 1}\right\}$ may not be almost surely constant; and for $d=1$ or 2 the limit process is either nondegenerate or totally degenerate (i.e. $C=0$ ) and $\left\{A_{n, 1}\right\}$ may have almost surely constant weak limit points; total degeneracy occurs if and only if $\tau_{1}$ and all weak limit points of $\left\{A_{n, 1}\right\}$ are almost surely constant.

If the matrices $A_{n, i}$ are such that, as functions of the direction $x$ the distributions of the projection of $A_{n, 2} x$ on $x$ and of the projection of $A_{n, 3}^{\prime} A_{n, 2} x$ on $x$ are invariant under the random transformation $A_{n, 1}$, or if the matrices $A_{n, 1}$ satisfy the condition

$$
E\left(A_{n, 1}^{\prime}\left(E A_{n, 1}+E A_{n, 1}^{\prime}\right) A_{n, 1}\right)=E A_{n, 1}+E A_{n, 1}^{\prime}
$$

and

$$
E\left(A_{n, 1}^{\prime}\left(E A_{n, 1}^{\prime} E A_{n, 1}\right) A_{n, 1}\right)=E A_{n, 1}^{\prime} E A_{n, 1},
$$

which is a consequence of the above property, then the limit process is a $d$-dimensional Brownian motion process with variance parameter

$$
\frac{1}{d E \tau_{1}}\left(\operatorname{Var} \tau_{1}+\left(E \tau_{1}\right)^{2} \lim _{n \rightarrow \infty} \frac{1-\sum_{j=1}^{d}\left(E a_{j i}^{(n)}\right)^{2}}{1-2 E a_{i i}^{(n)}+\sum_{j=1}^{d}\left(E a_{j i}^{(n)}\right)^{2}}\right),
$$

where $A_{n, 1}=\left(a_{i j}^{(n)}\right)$ and $i$ is arbitrary. This is always the case for $d=1$ or 2 . 
This result includes central limit theorems for the plane, with special distributions of the random times and direction changes, that have been obtained using methods different from ours by M. Kac [2, pp. 25-33], V. N. Tutubalin [5], and T. Watanabe [6].

As to the proof, in order to apply known results from the theory of weak convergence it was necessary to develop various renewal theoretic techniques, and to obtain limit theorems for sums of products of independent identically distributed irreducible random orthogonal matrices. It is these limit theorems that made possible the proof for any dimension $\geqq 3$. The weak convergence of the one (time)-dimensional distributions of $X_{n} / a_{n}$ to those of $X$ is obtained by using a theorem of B. Rosén [4], and it is shown that the convergence of all the finite-dimensional distributions follows from the convergence of the one-dimensional distributions. The applications of Rosén's theorem involves the above-mentioned limit theorems, and bounds for the distributions of suprema of the increments of $X_{n}$, which were obtained by using inequalities due to P. Billingsley [1, \$12]; these bounds also imply the tightness of the sequence $\left\{X_{n} / a_{n}\right\}$. The passage from the convergence on finite time intervals to the convergence on the semi-infinite time interval is justified by the result of C. Stone [3].

\section{REFERENCES}

1. P. Billingsley, Convergence of probability measures, Wiley, New York, 1968. MR 38 \# 1718 .

2. M. Kac, Probability and related topics in physical sciences, Lectures in Appl. Math., vol. 1, Interscience, New York, 1959. MR 21 \# 1635.

3. C. Stone, Weak convergence of stochastic processes defined on semi-infinite time intervals, Proc. Amer. Math. Soc. 14 (1963), 694-696. MR 27 \# 3015.

4. B. Rosén, On asymptotic normality of sums of dependent random vectors, $Z$. Wahrscheinlichkeitstheorie und Verw. Gebiete 7 (1967), 95-102. MR 35 \# 1074.

5. V. N. Tutubalin, The central limit theorem for random motions of Euclidean space, Vestnik Moskov. Univ. Ser. I Mat. Meh. 22 (1967), no. 6, 100-108. (Russian) MR 39 \# 3566.

6. T. Watanabe, Weak convergence of the isotropic scattering transport process with one speed in the plane to Brownian motion, Proc. Japan Acad. 44 (1968), 677-680. MR 38 \# 5289.

Department of Mathematics, Centro de Investigacion y de Estudios Avanzados, Instituto Politecnico Nacional, Mexico 14, D.F., MeXico 